CERN-TH/98-278

ETH-TH/98-24

GEF-TH-9/1998

IFUM 630/FT

hep-ph/9809367

\title{
Problems IN THE RESUMMATION OF SOFT-GLUON EFFECTS IN THE TRANSVERSE-MOMENTUM DISTRIBUTIONS OF MASSIVE VECTOR BOSONS IN HADRONIC COLLISIONS
}

\author{
Stefano Frixione円 \\ Theoretical Physics, ETH, Zurich, Switzerland \\ Paolo Nason ${ }^{\text {阝 }}$ \\ TH Division, CERN, Geneva, Switzerland

\section{Giovanni Ridolfi} \\ INFN, Sezione di Genova, Genoa, Italy
}

\begin{abstract}
We consider the resummation of soft-gluon emission in the transverse-momentum distribution of vector mesons in hadronic collisions. We find that the resummed expression in the impact-parameter formulation has an expansion in $\alpha_{s}$ with factorially growing terms with oscillating signs. These diverging terms arise from the small impact-parameter region of integration, and are of a subleading nature. We also obtain a closed expression for the next-to-leading logarithm resummation in $q_{\mathrm{T}}$-space, and we study its analytic structure. We find in this case that, although no factorially growing terms are present, there are geometrical singularities that severely restrict the range of applicability of the resummation formula.
\end{abstract}

CERN-TH/98-278

September 1998

\footnotetext{
${ }^{1}$ Work supported by the Swiss National Foundation.

${ }^{2}$ Address after October $1^{\text {st }}$, 1998: TH Division, CERN, Geneva, Switzerland.

${ }^{3}$ On leave of absence from INFN, Milan, Italy.
} 


\section{Introduction}

In this paper we discuss soft-gluon resummation effects in the transverse momentum distributions of heavy systems produced in hadronic collisions. We will mainly focus on the Drell-Yan process, but several aspects of the problems we are considering here are of a general nature, and apply also to other cases, such as the production of Higgs bosons or of heavy-quark pairs.

To simplify the notation, we consider the production of a virtual photon of offshellness $Q$ (this case can be trivially extended to $W$ or $Z$ production). We consider the kinematic region where its transverse momentum $q_{\mathrm{T}}$ is much less than $Q$, but much larger than $\Lambda_{\mathrm{QCD}}$. This regime, characterized by two distinct scales, has been extensively studied in the past [四, 2]. Large logarithms of the ratio $Q / q_{\mathrm{T}}$ (the socalled Sudakov logarithms) arise to all orders of the perturbative expansion, with up to two powers of the logarithm for each power of $\alpha_{s}$. All-order resummation of the perturbative expansion is needed in order to make sensible predictions in this regime. In the small- $q_{\mathrm{T}}$ limit, the resummed cross section has the structure suggested by Dokshitzer, Dyakonov and Troyan (DDT) in ref. [1]:

$$
\begin{aligned}
\frac{d \sigma}{d Q^{2} d q_{\mathrm{T}}^{2}}= & \frac{\sigma_{0}}{Q^{2}} \sum_{q} e_{q}^{2} \frac{d}{d q_{\mathrm{T}}^{2}} \int d x_{\mathrm{A}} d x_{\mathrm{B}} \delta\left(x_{\mathrm{A}} x_{\mathrm{B}}-\tau\right) \\
& \times\left\{f_{q / \mathrm{A}}\left(x_{\mathrm{A}}, q_{\mathrm{T}}\right) f_{\bar{q} / \mathrm{B}}\left(x_{\mathrm{B}}, q_{\mathrm{T}}\right) \exp \tilde{\mathcal{S}}\left(Q, q_{\mathrm{T}}\right)+(q \leftrightarrow \bar{q})\right\},
\end{aligned}
$$

where $\sigma_{0}=4 \pi \alpha_{e m}^{2} /(9 s)$ is the lowest-order cross section for the process $q \bar{q} \rightarrow \mu^{+} \mu^{-}$, $\tau=Q^{2} / s, s$ is the hadronic centre-of-mass energy squared, and

$$
\tilde{\mathcal{S}}\left(Q, q_{\mathrm{T}}\right)=-\int_{q_{\mathrm{T}}^{2}}^{Q^{2}} \frac{d \mu^{2}}{\mu^{2}}\left[\tilde{A}\left(\alpha_{s}\left(\mu^{2}\right)\right) \log \frac{Q^{2}}{\mu^{2}}+\tilde{B}\left(\alpha_{s}\left(\mu^{2}\right)\right)\right]
$$

with

$$
\tilde{A}\left(\alpha_{s}\right)=\tilde{A}_{1} \alpha_{s}+\tilde{A}_{2} \alpha_{s}^{2}+\ldots, \quad \tilde{B}\left(\alpha_{s}\right)=\tilde{B}_{1} \alpha_{s}+\ldots .
$$

In eq. (1.1), the leading logarithmic (LL) level of accuracy, which accounts for all terms of order $\alpha_{s}^{n} l^{n+1}$ (where $l=\log \left(Q^{2} / q_{\mathrm{T}}^{2}\right)$ ) in eq. (1.2), is achieved by including only the $\tilde{A}_{1}$ term of the expansion of $\tilde{A}\left(\alpha_{s}\right)$. The next-to-leading logarithmic (NLL) level, which amounts to the inclusion of all terms of order $\alpha_{s}^{n} l^{n}$, is achieved if one includes also the $\tilde{A}_{2}$ and the $\tilde{B}_{1}$ terms. Using $q_{\mathrm{T}}$ instead of $Q$ in the parton densities 
also corresponds to a NLL correction.

We observe that no simple classification of the Sudakov logarithms can be given by looking at the expansion of the cross section (1.1) in powers of $\alpha_{s}$ and of $l$. This is because the exponent $\tilde{\mathcal{S}}$ has the formal expansion in $\alpha_{s}$

$$
\tilde{\mathcal{S}}\left(Q, q_{\mathrm{T}}\right)=\sum_{n=1}^{\infty} d_{n, n+1} \alpha_{s}^{n}\left(Q^{2}\right) l^{n+1}+\sum_{n=1}^{\infty} d_{n, n} \alpha_{s}^{n}\left(Q^{2}\right) l^{n}+\ldots
$$

When only LL terms are included, the neglected NLL corrections correspond to a factor, which is a power series in terms of $\alpha_{s} l$, with the $0^{\text {th }}$ order term equal to 1 . Thus, as long as $\alpha_{s} l<1$, the expansion is valid. On the other hand, by expanding the exponential of the first LL term, which is of order $\alpha_{s} l^{2}$, we can get arbitrarily large powers of $\alpha_{s} l^{2}$, and NLL corrections would appear to be of the order of $\alpha_{s}^{n} l^{2 n-1}$. In this case, the expansion would seem to be valid only when $\alpha_{s} l^{2}<1$, a much more restricted range.

As we will show later, eq. (1.1) has never appeared in the literature in a complete form at the NLL level. Indeed, it was shown in ref. [2] that the resummation of soft-gluon effects is most naturally performed using the impact-parameter formalism. A general formula for the Drell-Yan cross section in the impact-parameter space has been obtained in ref. [3]:

$$
\begin{aligned}
& \frac{d \sigma}{d Q^{2} d q_{\mathrm{T}}^{2}}=\frac{\sigma_{0}}{4 \pi Q^{2}} \sum_{q} e_{q}^{2} \int d x_{\mathrm{A}} d x_{\mathrm{B}} \delta\left(x_{\mathrm{A}} x_{\mathrm{B}}-\tau\right) \int d^{2} b e^{i \vec{q}_{\mathrm{T}} \cdot \vec{b}} \\
& \quad \times\left\{f_{q / \mathrm{A}}\left(x_{\mathrm{A}}, c_{1} / b\right) f_{\bar{q} / \mathrm{B}}\left(x_{\mathrm{B}}, c_{1} / b\right) \exp \mathcal{S}(Q, b)+(q \leftrightarrow \bar{q})\right\},
\end{aligned}
$$

where

$$
\begin{aligned}
& \mathcal{S}(Q, b)=-\int_{c_{1}^{2} / b^{2}}^{c_{2}^{2} Q^{2}} \frac{d \mu^{2}}{\mu^{2}}\left[A\left(\alpha_{s}\left(\mu^{2}\right)\right) \log \frac{c_{2}^{2} Q^{2}}{\mu^{2}}+B\left(\alpha_{s}\left(\mu^{2}\right)\right)\right] \\
& A\left(\alpha_{s}\right)=A_{1} \alpha_{s}+A_{2} \alpha_{s}^{2}+\ldots, \quad B\left(\alpha_{s}\right)=B_{1} \alpha_{s}+\ldots,
\end{aligned}
$$

and $c_{1}, c_{2}$ are arbitrary constants of order one. The coefficients $A_{i}$ and $B_{i}$ also carry a dependence upon these arbitrary constants (see Appendix A). There are well-known

\footnotetext{
${ }^{4}$ In order to go beyond the NLL terms, besides the inclusion of higher-order terms in the expansion of $\tilde{A}\left(\alpha_{s}\right)$ and $\tilde{B}\left(\alpha_{s}\right)$, it is also needed to perform a suitable change of scheme on the parton densities.

${ }^{5}$ In ref. [3], eq. (1.5) is claimed to be valid also for $q_{\mathrm{T}}<\Lambda_{\mathrm{QCD}}$. This regime is not under investigation here.
} 
problems that arise when applying eq. (1.5) to obtain phenomenological results. First of all, one can immediately recognize that, for any value of $q_{\mathrm{T}}$, eq. (1.5), as it stands, is undefined, because the $b$ integration forces the scale at which $\alpha_{s}$ and the parton densities are evaluated, to approach the non-perturbative region. This problem is usually avoided by replacing $b$ in $\mathcal{S}(Q, b)$ and in the parton densities with [4]

$$
b^{\star}=\frac{b}{\sqrt{1+\left(b / b_{\lim }\right)^{2}}},
$$

which approaches $b$ for $b \ll b_{\text {lim }}$, and never exceeds $b_{\text {lim }}$ for large values of $b$. Choosing $b_{\lim }$ of the order of $1 \mathrm{GeV}^{-1}$, one thus avoids the non-perturbative region. This procedure introduces corrections of the order of powers of $1 / b_{\lim }$ to eq. (1.5). Apart from the introduction of $b^{\star}$, eq. (1.5) is usually also supplemented with a non-perturbative correction factor of the form $\exp \left(-g b^{2}\right)$. We stress that this factor is actually not needed in order for the integral in eq. (1.5) to converge (a proof is given in Appendix D).

In the present work, we will obtain an explicit expression for eq. (1.1), valid at the NLL level, and we will address several theoretical problems in the formulation of eqs. (1.1) and (1.5).

\section{$2 \quad q_{\mathrm{T}}$-space formulation of the resummed cross section}

In this section, we will obtain a $q_{\mathrm{T}}$-space expression for the cross section given in eq. (1.5), which is valid in the NLL approximation. We will analitycally perform the integration over $b$, which appears in eq. (1.5). We will retain only the terms required for maintaining a NLL accuracy in the formal power expansion of the cross section. In other words, we will obtain an expansion for $\tilde{\mathcal{S}}\left(Q, q_{\mathrm{T}}\right)$ that corresponds to retaining the first two sums of eq. (1.4), and nothing more. We will first consider a simplified model, which corresponds roughly to the assumption of scale-independent parton densities and coupling constant. This model can be worked out quite easily, and it displays some features and problems that persist in the realistic case.

\footnotetext{
${ }^{6}$ In fact, it is argued in ref. [3] that the non-perturbative region contributes a factor of the form $\exp \left[-g_{1}(b) \log \left(Q^{2}\right)-g_{2}(b)\right]$.
} 


\section{$2.1 \quad$ A simple model}

We thus assume that the parton densities and $\alpha_{s}$ do not depend on the energy scale. In this approximation, eq. (1.5) factorizes in a simple form:

$$
\frac{d \sigma}{d Q^{2} d q_{\mathrm{T}}^{2}}=\frac{\sigma_{0}}{Q^{4}} F\left(Q, q_{\mathrm{T}}\right) \sum_{q} e_{q}^{2} \int d x_{\mathrm{A}} d x_{\mathrm{B}} f_{q / \mathrm{A}}\left(x_{\mathrm{A}}\right) f_{\bar{q} / \mathrm{B}}\left(x_{\mathrm{B}}\right) \delta\left(x_{\mathrm{A}} x_{\mathrm{B}}-\tau\right),
$$

where, following ref. [5], we have defined the form factor

$$
F\left(Q, q_{\mathrm{T}}\right)=\frac{Q^{2}}{4 \pi} \int d^{2} b e^{i \vec{q}_{\mathrm{T}} \cdot \vec{b}} \exp \mathcal{S}(Q, b) .
$$

After performing the angular integration in eq. (2.2) and changing the integration variable from $b$ to $\hat{b}=b q_{\mathrm{T}}$ we get

$$
F\left(Q, q_{\mathrm{T}}\right)=\frac{Q^{2}}{2 q_{\mathrm{T}}^{2}} \int_{0}^{\infty} d \hat{b} \hat{b} J_{0}(\hat{b}) \exp \mathcal{S}\left(Q, \hat{b} / q_{\mathrm{T}}\right)
$$

where $J_{0}$ is the $0^{\text {th }}$-order Bessel function of the first kind. Equation (2.3) can be integrated by parts, exploiting the identity

$$
x J_{0}(x)=\frac{d}{d x}\left(x J_{1}(x)\right) .
$$

We obtain

$$
F\left(Q, q_{\mathrm{T}}\right)=Q^{2} \frac{d}{d q_{\mathrm{T}}^{2}} \int_{0}^{\infty} d \hat{b} J_{1}(\hat{b}) \exp \mathcal{S}\left(Q, \hat{b} / q_{\mathrm{T}}\right) .
$$

In this way, we have an expression in the DDT form, analogous to eq. (1.1).

We assume that the Sudakov form factor at the LL level takes the form

$$
\mathcal{S}\left(Q, \hat{b} / q_{\mathrm{T}}\right)=-a L^{2}
$$

where

$$
L=\log \frac{Q^{2} \hat{b}^{2}}{q_{\mathrm{T}}^{2}}
$$

and

$$
a=\frac{A_{1}}{2} \alpha_{s}
$$

which is what is obtained if the running of $\alpha_{s}$ is neglected in eq. (1.6). In this context we take the arbitrary constants $c_{1}, c_{2}$ to be equal to 1 ; different choices amount to a rescaling of the coupling constant. Defining

$$
l=\log \frac{Q^{2}}{q_{\mathrm{T}}^{2}}, \quad l_{\hat{b}}=\log \hat{b}^{2},
$$


we obtain

$$
F\left(Q, q_{\mathrm{T}}\right)=Q^{2} \frac{d}{d q_{\mathrm{T}}^{2}}\left\{\exp \left(-a l^{2}\right) \int_{0}^{\infty} d \hat{b} J_{1}(\hat{b}) \exp \left(-2 a l l_{\hat{b}}-a l_{\hat{b}}^{2}\right)\right\} .
$$

The exponential under the integral sign can now be expanded and integrated term by term. In fact, $J_{1}(\hat{b})$ has an oscillating behaviour for large $\hat{b}$, which gives rise to finite integrals when multiplied by any power of $l_{\hat{b}}$. Furthermore, all terms of the expansion will have at most as many powers of $l$ as powers of $a$. The NLL level of accuracy is reached by neglecting the $a l_{\hat{b}}^{2}$ term in the exponent, which is formally subleading. We thus get, at the NLL level,

$$
\begin{aligned}
F\left(Q, q_{\mathrm{T}}\right) & =Q^{2} \frac{d}{d q_{\mathrm{T}}^{2}}\left[\exp \left(-a l^{2}\right) \int_{0}^{\infty} d \hat{b} J_{1}(\hat{b}) \hat{b}^{-4 a l}\right] \\
& =Q^{2} \frac{d}{d q_{\mathrm{T}}^{2}}\left[\exp \left(-a l^{2}\right) 2^{-4 a l} \frac{\Gamma(1-2 a l)}{\Gamma(1+2 a l)}\right]
\end{aligned}
$$

where the last integral was performed using the result

$$
\int_{0}^{\infty} d x x^{h} J_{1}(x)=2^{h} \frac{\Gamma(1+h / 2)}{\Gamma(1-h / 2)}, \quad-2<h<\frac{1}{2} .
$$

The form factor in eq. (2.11) is singular for $a l \rightarrow 1 / 2$. Considered as a function of $a$ at a given value of $l$, it is analytic in a circle of radius $1 /(2 l)$ around $a=0$. Thus, when expanded in powers of $a$, the coefficients of the expansion grow at most geometrically.

On the other hand, the original expression eq. (2.10) is well defined for any value of $a>0$ and it is divergent for any negative value of $a$. Thus it cannot be analytic in the neighbourhood of the origin. This observation anticipates our conclusions: the original form factor has a divergent power series expansion in $a$, but, if we retain only the LL and NLL terms, we are left with a convergent expansion. Thus, the divergent behaviour must be ascribed to the subleading terms in the original expression.

We now analyse more closely the origin of the non-analyticity of eq. (2.10), now interpreted as defining a formal expansion in powers of $a$ (it is easy to see that the coefficients of this expansion are finite order by order). First of all, we separate the large- $\hat{b}$ and small- $\hat{b}$ contributions to the integral. No singularities may arise from the large- $\hat{b}$ region. This can be shown as follows. We rewrite the large $-\hat{b}$ part of the integral in eq. (2.10) as

$$
\int_{\hat{b}_{0}}^{\infty} d \hat{b} J_{1}(\hat{b}) \exp \left(-2 a l l_{\hat{b}}-a l_{\hat{b}}^{2}\right)=-\frac{2}{\pi} \operatorname{Re}\left[\int_{\hat{b}_{0}}^{\infty} d \hat{b} K_{1}(-i \hat{b}) \exp \left(-2 a l l_{\hat{b}}-a l_{\hat{b}}^{2}\right)\right],
$$


where $\hat{b}_{0}$ is an arbitrary cut-off. We can now deform the integration contour counterclockwise, letting $\hat{b} \rightarrow i \hat{b}$. Because of the asymptotic behaviour

$$
K_{1}(\hat{b}) \approx \sqrt{\frac{\pi}{2 \hat{b}}} e^{-\hat{b}}
$$

the integral is strongly convergent, with all its derivatives with respect to $a$, regardless of the sign of $a$. It thus defines an analytic function with infinite radius of convergence.

We now study the small- $\hat{b}$ contribution to the integral. Using the small- $\hat{b}$ behaviour $J_{1}(\hat{b}) \approx \hat{b} / 2$, we get

$$
\int_{0}^{\hat{b}_{0}} d \hat{b} \hat{b} \exp \left(-2 a l l_{\hat{b}}-a l_{\hat{b}}^{2}\right)=\frac{\hat{b}_{0}^{2}}{2} e^{a l^{2}} \sum_{n=0}^{\infty} a^{n} \sum_{i=0}^{2 n}(-1)^{n-i} \frac{(2 n) !}{n ! i !}\left(l+l_{\hat{b}_{0}}\right)^{i} .
$$

We see that subleading terms in this expansion have factorially growing coefficients. For example, the terms corresponding to $i=0$ grow as $(2 n) ! / n !$. The factorial growth is accompanied by sign oscillations. This is why, when $a>0$, this asymptotic expansion corresponds to a finite expression.

In summary, we started with an expression that had an essential singularity at $a=$ 0 , arising from the small- $\hat{b}$ region of integration, but well defined for all positive values of $a$. By dropping all terms beyond the leading and next-to-leading, we obtained an expression that is analytic around the origin, but with poles at positive, finite values of $a$. We should now give our judgement, and decide which one of the two expressions is the most reliable. It is clear from the start that the factorially growing terms in our initial expression have no justification. It has been pointed out [6] that, in fact, the expression for the Sudakov form factor should carry a theta function

$$
\mathcal{S}(Q, b) \rightarrow \theta(b-1 / Q) \mathcal{S}(Q, b)
$$

since no large logarithms of $b$ arise from the small- $b$ region in the exact calculation of the first-order correction to the Drell-Yan cross section. This theta function would suppress the integrand in the region $0<\hat{b}<q_{\mathrm{T}} / Q$. It would therefore amount to a power-suppressed correction that removes the small- $\hat{b}$ singularities. In fact

$$
\int_{0}^{\infty} e^{-a L^{2} \theta\left(\hat{b}-q_{\mathrm{T}} / Q\right)} J_{1}(\hat{b}) d \hat{b}=\int_{0}^{\infty} e^{-a L^{2}} J_{1}(\hat{b}) d \hat{b}-\int_{0}^{q_{\mathrm{T}} / Q}\left[e^{-a L^{2}}-1\right] J_{1}(\hat{b}) d \hat{b} .
$$

If we expand the exponential in the first term, and integrate term by term, we find a power expansion in $a$ whose coefficients are polynomials in $\log q_{\mathrm{T}} / Q$. For the second 
term, the same procedure yields an expansion whose coefficients are suppressed by at least two powers of $q_{\mathrm{T}} / Q$, as can be seen by expanding the function $J_{1}$ for small values of its argument. The factorial growth present in the second term compensates the one present in the first one. The fact remains, however, that if we drop all power-suppressed effects from our expression, the factorial growth remains.

It should also be pointed out that the replacement given in eq. (2.16) is actually justified only for the first-order expansion of the Sudakov exponential. In fact, factorization of soft-gluon emission has a double origin. It comes from the dynamical factorization of the soft-gluon emission amplitudes, and from the kinematical factorization of the phase space for the emitted gluons. In the computation of the transverse momentum of DY pairs, the phase space factorizes naturally in the impact-parameter (i.e. in $b$ ) representation. However, neither the phase space nor the emission amplitudes factorize for the emission of hard gluons. To be more specific, while it seems reasonable to assume that gluon emission is cut off for gluons with transverse momentum larger than $Q$ (i.e. for $b<1 / Q$ ), for two-gluon emission this constraint should become stronger, something of the form $\left|q_{\mathrm{T} 1}\right|+\left|q_{\mathrm{T} 2}\right|<Q$, and so on. It is therefore quite possible that the factorial growth coming from the small- $\hat{b}$ region is only due to an improper treatment of the multigluon emission for large transverse momenta. Since the factorial growth is accompanied by sign alternation, it leads to a suppression of the small- $\hat{b}$ region, thus giving a more stable result, which is however not fully justified.

It is interesting to compare what we have found here with what was found in the case of resummation of threshold-enhanced Sudakov effects [7]. In that case, it had been found that factorial growth of the perturbative expansion was present in the $x$-space formulation of the resummation. Its origin is in the small-momentum region, and is caused by the fact that momentum conservation is violated in the $x$ space formalism. If one instead uses the Mellin transform (i.e. $N$-space) formalism, which correctly implements momentum conservation, no factorial growth is present. Kinematic factorization of soft-gluon emission is present in this case in the $N$-space formulation. It then turns out that no factorial growth is present when the resummation is performed in the most natural formalism.

In the case we are considering here, instead, the factorial growth arises in the most natural formulation, which is the $b$-space formulation. However, it does arise from the large-momentum region, which is unreliably treated by the resummation formalism. 
It is generally believed that factorial growth in the perturbative expansion may arise from ultraviolet or infrared renormalons. In the present case, as in the case of ref. [7], the factorial growth does not arise from renormalons, since it is present also at fixed $\alpha_{s}$. In both cases, the factorial growth appears to be an artefact of the formulation. This is reassuring, since in most studies of power-suppressed effects it is assumed that no other sources of factorial growth exist besides renormalons and instantons.

\subsection{The realistic case}

The realistic case is more complicated, because the parton densities carry a dependence on the scale $1 / b$, which prevents from factorizing the cross section as in eq. (2.1). We therefore proceed as in ref. [5] and take the Mellin transform with respect to $\tau$ (at fixed $Q^{2}$ ) of eq. (1.5):

$$
\sigma_{N}=\int_{0}^{1} d \tau \tau^{N-1} \frac{Q^{2}}{\sigma_{0}} \frac{d \sigma}{d Q^{2} d q_{\mathrm{T}}^{2}}
$$

We thus find

$$
\sigma_{N}=\frac{1}{4 \pi} \int d^{2} b e^{i \vec{q}_{\mathrm{T}} \cdot \vec{b}} C_{N}\left(c_{1} / b\right) \exp \mathcal{S}(Q, b)
$$

where

$$
C_{N}(\mu)=\sum_{q} e_{q}^{2} \int_{0}^{1} d x_{\mathrm{A}} x_{\mathrm{A}}^{N-1} f_{q / \mathrm{A}}\left(x_{\mathrm{A}}, \mu\right) \int_{0}^{1} d x_{\mathrm{B}} x_{\mathrm{B}}^{N-1} f_{\bar{q} / \mathrm{B}}\left(x_{\mathrm{B}}, \mu\right) .
$$

We now use the parton-density evolution equation to write]

$$
C_{N}\left(c_{1} / b\right)=C_{N}\left(c_{2} Q\right) \exp \mathcal{G}_{N}(Q, b)
$$

with

$$
\mathcal{G}_{N}(Q, b)=-2 \int_{c_{1}^{2} / b^{2}}^{c_{2}^{2} Q^{2}} \frac{d \mu^{2}}{\mu^{2}} \gamma_{N}\left(\alpha_{s}\left(\mu^{2}\right)\right) .
$$

The anomalous dimensions of the parton-density functions have a perturbative expansion given by

$$
\gamma_{N}\left(\alpha_{s}\right)=\gamma_{1, N} \alpha_{s}+\gamma_{2, N} \alpha_{s}^{2}+\ldots
$$

We have therefore

$$
\sigma_{N}=\frac{1}{Q^{2}} C_{N}\left(c_{2} Q\right) F_{N}\left(Q, q_{\mathrm{T}}\right)
$$

\footnotetext{
${ }^{7}$ In order to simplify the discussion, we only consider the non-singlet case here.
} 
where

$$
F_{N}\left(Q, q_{\mathrm{T}}\right)=\frac{Q^{2}}{4 \pi} \int d^{2} b e^{i \vec{q}_{\mathrm{T}} \cdot \vec{b}} \exp \mathcal{S}(Q, b) \exp \mathcal{G}_{N}(Q, b) .
$$

The form factor $F_{N}\left(Q, q_{\mathrm{T}}\right)$ can be computed in the same way as $F\left(Q, q_{\mathrm{T}}\right)$ in eq. (2.2). We thus obtain the analogue of eq. (2.5):

$$
F_{N}\left(Q, q_{\mathrm{T}}\right)=Q^{2} \frac{d}{d q_{\mathrm{T}}^{2}} \int_{0}^{\infty} d \hat{b} J_{1}(\hat{b}) \exp \mathcal{S}\left(Q, \hat{b} / q_{\mathrm{T}}\right) \exp \mathcal{G}_{N}\left(Q, \hat{b} / q_{\mathrm{T}}\right)
$$

In this case, however, the integrand in eq. (2.26) is not defined at large $\hat{b}$. In fact, for large $\hat{b}$ the lower integration bound in eqs. (1.6) and (2.22) decreases and eventually, for some value of $\hat{b}, \alpha_{s}\left(\mu^{2}\right)$ in the integrand is computed at the Landau pole. For the moment, we consider eq. (2.26) as a representation of its formal power expansion in $\alpha_{s}\left(Q^{2}\right)$. The Landau pole contributes to spoil the convergence of this expansion. We will study its convergence properties in Appendix $\mathrm{Q}$.

From eqs. (1.6) and (1.7) we can obtain an explicit expansion for $\mathcal{S}(Q, b)$, which turns out to have the form

$$
\mathcal{S}(Q, b)=\sum_{i=0}^{\infty} \alpha_{s}^{i-1} f_{i}\left(\alpha_{s} L\right), \quad \alpha_{s}=\alpha_{s}\left(c_{2}^{2} Q^{2}\right), \quad L=\log \frac{c_{2}^{2} Q^{2} \hat{b}^{2}}{c_{1}^{2} q_{\mathrm{T}}^{2}}
$$

In the following we will consider the NLL expansion (in terms of $L$ ), which corresponds to

$$
\mathcal{S}(Q, b) \simeq \mathbb{S}\left(\alpha_{s}, L\right) \equiv \frac{1}{\alpha_{s}} f_{0}\left(\alpha_{s} L\right)+f_{1}\left(\alpha_{s} L\right)
$$

where

$$
\begin{aligned}
f_{0}(y)= & \frac{A_{1}}{b_{0}^{2}}\left[b_{0} y+\log \left(1-b_{0} y\right)\right] \\
f_{1}(y)= & \frac{A_{1} b_{1}}{b_{0}^{2}}\left[\frac{1}{2} \log ^{2}\left(1-b_{0} y\right)+\frac{b_{0} y}{1-b_{0} y}+\frac{\log \left(1-b_{0} y\right)}{1-b_{0} y}\right] \\
& -\frac{A_{2}}{b_{0}^{2}}\left[\log \left(1-b_{0} y\right)+\frac{b_{0} y}{1-b_{0} y}\right]+\frac{B_{1}}{b_{0}} \log \left(1-b_{0} y\right),
\end{aligned}
$$

and $b_{0}, b_{1}$ are the first coefficients of the QCD $\beta$ function:

$$
\begin{aligned}
& \mu^{2} \frac{\partial \alpha_{s}\left(\mu^{2}\right)}{\partial \mu^{2}}=-b_{0} \alpha_{\mathrm{S}}^{2}\left(1+b_{1} \alpha_{s}+\ldots\right), \\
& b_{0}=\frac{33-2 n_{f}}{12 \pi} ; \quad b_{1}=\frac{1}{2 \pi} \frac{153-19 n_{f}}{33-2 n_{f}} .
\end{aligned}
$$


Observe that $f_{0}(y)$ is of order $y^{2}$, and $f_{1}(y)$ is of order $y$ as $y \rightarrow 0$. We expect the remaining $f_{i}(y)$ to have a power expansion in $y$ around $y=0$.

Equation (2.27) represents a logarithm expansion of the form factor $\mathcal{S}$ in terms of $L$. However, as previously observed, the large logarithm of our physical problem is $l$ rather than $L$, which depends on the integration variable $\hat{b}$. The leading and next-to-leading logarithm expansions of the form factor $\mathcal{S}$ in terms of $l$ are

$$
\begin{aligned}
\mathcal{S}_{\mathrm{LL}}\left(Q, \hat{b} / q_{\mathrm{T}}\right) & =\frac{1}{\alpha_{s}} f_{0}\left(\alpha_{s} l\right), \\
\mathcal{S}_{\mathrm{NLL}}\left(Q, \hat{b} / q_{\mathrm{T}}\right) & =\frac{1}{\alpha_{s}} f_{0}\left(\alpha_{s} l\right)+f_{1}\left(\alpha_{s} l\right)+\frac{d f_{0}\left(\alpha_{s} l\right)}{d\left(\alpha_{s} l\right)} l_{\hat{b}} .
\end{aligned}
$$

On the other hand, $\mathcal{G}_{N}$ is a pure NLL term, since the scale dependence of parton densities gives rise to corrections, which have the form of a power series in $\alpha_{s} l$. Furthermore, from the definition given in eq. (2.22), we have

$$
\mathcal{G}_{N}\left(Q, \hat{b} / q_{\mathrm{T}}\right)=\mathcal{G}_{N}\left(Q, 1 / q_{\mathrm{T}}\right)+\text { NNLL terms. }
$$

We first consider the LL approximation. In this case the $\hat{b}$ integration is trivial, because $\exp \mathcal{S}$ is independent of $\hat{b}$ at the LL level, as is clear from eq. (2.33). The factor in eq. (2.22) being a pure NLL correction, we therefore find

$$
F_{N}\left(Q, q_{\mathrm{T}}\right)=Q^{2} \frac{d}{d q_{\mathrm{T}}^{2}} \exp \mathcal{S}_{\mathrm{LL}}
$$

which is, up to NLL terms, the result of ref. [5].

We now turn to the NLL approximation. Using eq. (2.34) we have

$$
\exp \mathcal{S}_{\mathrm{NLL}}\left(Q, \hat{b} / q_{\mathrm{T}}\right)=\left(\frac{c_{2} \hat{b}}{c_{1}}\right)^{h} \exp \mathbb{S}\left(\alpha_{s}, l\right)
$$

where

$$
h=2 \frac{d f_{0}\left(\alpha_{s} l\right)}{d\left(\alpha_{s} l\right)}=-\frac{2 A_{1}}{b_{0}} \frac{b_{0} \alpha_{s} l}{1-b_{0} \alpha_{s} l} .
$$

Since at NLL $\mathcal{G}_{N}$ is independent of $\hat{b}$, we have

$$
\begin{gathered}
F_{N}\left(Q, q_{\mathrm{T}}\right)=Q^{2} \frac{d}{d q_{\mathrm{T}}^{2}}\left[\exp \mathcal{G}_{N}\left(Q, 1 / q_{\mathrm{T}}\right) \exp \mathbb{S}\left(\alpha_{s}, l\right)\left(\frac{c_{2}}{c_{1}}\right)^{h} \int_{0}^{\infty} d \hat{b} \hat{b}^{h} J_{1}(\hat{b})\right] \\
=Q^{2} \frac{d}{d q_{\mathrm{T}}^{2}}\left[\exp \mathcal{G}_{N}\left(Q, 1 / q_{\mathrm{T}}\right) \exp \mathbb{S}\left(\alpha_{s}, l\right)\left(\frac{2 c_{2}}{c_{1}}\right)^{h} \frac{\Gamma(1+h / 2)}{\Gamma(1-h / 2)}\right] .
\end{gathered}
$$


Equation (2.39) represents our final result for the form factor $F_{N}\left(Q, q_{\mathrm{T}}\right)$ at NLL. It can be used to obtain an expression similar to eq. (1.1) for the cross section; in fact, using the procedure outlined in ref. [5], we may replace eq. (2.39) in eq. (2.19) and obtain

$$
\begin{aligned}
\sigma_{N} & =\frac{d}{d q_{\mathrm{T}}^{2}}\left[C_{N}\left(c_{2} Q\right) \exp \mathcal{G}_{N}\left(Q, 1 / q_{\mathrm{T}}\right) \exp \mathbb{S}\left(\alpha_{s}, l\right)\left(\frac{2 c_{2}}{c_{1}}\right)^{h} \frac{\Gamma(1+h / 2)}{\Gamma(1-h / 2)}\right] \\
& =\frac{d}{d q_{\mathrm{T}}^{2}}\left[C_{N}\left(c_{1} q_{\mathrm{T}}\right) \exp \mathbb{S}\left(\alpha_{s}, l\right)\left(\frac{2 c_{2}}{c_{1}}\right)^{h} \frac{\Gamma(1+h / 2)}{\Gamma(1-h / 2)}\right]
\end{aligned}
$$

where we have used eq. (2.21). The $N$ dependence has been completely absorbed in the factor $C_{N}\left(c_{1} q_{\mathrm{T}}\right)$, so that the inverse Mellin transform can be performed immediately and the cross section takes the form

$$
\begin{aligned}
& \frac{d \sigma}{d Q^{2} d q_{\mathrm{T}}^{2}}=\frac{\sigma_{0}}{Q^{2}} \sum_{q} e_{q}^{2} \frac{d}{d q_{\mathrm{T}}^{2}} \int d x_{\mathrm{A}} d x_{\mathrm{B}} \delta\left(x_{\mathrm{A}} x_{\mathrm{B}}-\tau\right) \\
& \times\left\{f_{q / \mathrm{A}}\left(x_{\mathrm{A}}, q_{\mathrm{T}}\right) f_{\bar{q} / \mathrm{B}}\left(x_{\mathrm{B}}, q_{\mathrm{T}}\right) \exp \mathbb{S}\left(\alpha_{s}, l\right)\left(\frac{2 c_{2}}{c_{1}}\right)^{h} \frac{\Gamma(1+h / 2)}{\Gamma(1-h / 2)}+(q \leftrightarrow \bar{q})\right\},
\end{aligned}
$$

where $h$ is given in eq. (2.38). Equation (2.41) gives an explicit expression of the resummed transverse-momentum distribution of DY pairs at NLL accuracy. This result bears some similarities with that of ref. [8], but is in fact different. The difference arises because we insist on keeping leading and next-to-leading terms according to the classification described in the Introduction, while in ref. [8] the emphasis is on finding a good appoximation to the $b$-space result. A formula analogous to ours, but for the case of the energy-energy correlation in $e^{+} e^{-}$annihilations, can instead be found in ref. [9].

As in the case of the simple example treated in the previous subsection, our expression is analytic in $\alpha_{s}$ for small $\alpha_{s}$, and displays a pole at $h=-2$, which corresponds to

$$
\alpha_{s}=\frac{1}{l\left(A_{1}+b_{0}\right)} .
$$

This can also be seen as a lower limit on the allowable values of $q_{\mathrm{T}}$ :

$$
q_{\mathrm{T}}>Q \exp \left(-\frac{1}{2 \alpha_{s}\left(A_{1}+b_{0}\right)}\right) \simeq \Lambda_{\mathrm{QCD}}\left(\frac{Q}{\Lambda_{\mathrm{QCD}}}\right)^{\frac{A_{1}}{A_{1}+b_{0}}} .
$$


We thus find the disappointing result that our formula breaks down at a value of $q_{\mathrm{T}}$ that is an increasing function of $Q / \Lambda_{\mathrm{QCD}}$.

The full $b$-space expression is instead well defined for all positive values of $\alpha_{s}$. However, it is not analytic around the origin. We will now prove that the small- $\hat{b}$ region contributions to the coefficients of its power expansion are at least as divergent as in the simple example examined in the previous subsection. We thus consider the integral

$$
\int_{0}^{1} d \hat{b} \hat{b} \exp \left\{\frac{1}{\alpha_{s} b_{0}^{2}}\left[\alpha_{s} b_{0} L+\log \left(1-\alpha_{s} b_{0} L\right)\right]\right\}
$$

with $L=\log \left(Q^{2} \hat{b}^{2} / q_{\mathrm{T}}{ }^{2}\right)$, where, since we are considering the small- $\hat{b}$ region, we have approximated $J_{1}(\hat{b}) \approx \hat{b} / 2$. We have fixed for simplicity $\hat{b}_{0}=1$. We will focus on the most subleading terms, that is to say, those carrying no powers of $\log \left(q_{\mathrm{T}} / Q\right)$ in the expansion. These terms are obtained by simply taking $q_{\mathrm{T}}=Q$. Expanding the exponent in powers of $\alpha_{s}$, our integral becomes

$$
\begin{aligned}
& \int_{0}^{1} d \hat{b} \hat{b} \exp \left\{\frac{1}{b_{0}^{2}} \sum_{k=2}^{\infty} \frac{\left(-\alpha_{s}\right)^{k-1} b_{0}^{k}\left(-l_{\hat{b}}\right)^{k}}{k}\right\} \\
& =\int_{0}^{1} d \hat{b} \hat{b} \sum_{j=0}^{\infty} \frac{\left(-\alpha_{s}\right)^{j}}{j !}\left[\frac{1}{2^{j}}\left(-l_{\hat{b}}\right)^{2 j}+r_{1}\left(-l_{\hat{b}}\right)^{2 j-1}+\ldots\right],
\end{aligned}
$$

where the highest power in $l_{\hat{b}}$ comes from the exponentiation of the $k=2$ term in the exponent, whose power of $l_{\hat{b}}$ is twice the corresponding power of $\alpha_{s}$. The important thing to notice is that all the remaining coefficients $r_{1}, r_{2}$, etc., are positive. Thus each term of the sum is positive, and the value of each coefficient is bounded from below by the value of the first term $\int_{0}^{1} d \hat{b} \hat{b}\left(-l_{\hat{b}}\right)^{2 j}=(2 j) ! / 2$, which shows the presence of factorial growth.

In order to complete our task, we should show that no factorial growth arises from the large- $\hat{b}$ region of integration

$$
\int_{1}^{\infty} d \hat{b} J_{1}(\hat{b}) \exp \left\{\frac{1}{\alpha_{s} b_{0}^{2}}\left[\alpha_{s} b_{0} L+\log \left(1-\alpha_{s} b_{0} L\right)\right]\right\} .
$$

Unlike the case of the simple model, here we can no longer rely upon the analyticity in $\alpha_{s}$ of this integral. In fact, the logarithm in the exponent encounters a Landau pole at $\alpha_{s} b_{0} L=1$, and thus analyticity in $\alpha_{s}$ cannot possibly be there. Thus, the coefficients of its perturbative expansion will grow faster than any power, but it is still possible to show that they grow less than factorially, by using the fact that the 
above integral can be related to an exponentially dumped one. The demonstration, similar to the analogous one in ref. [7], is given in Appendix C.

\section{Comparison of various approaches}

We will now compare the numerical differences of the various approaches for the computation of the Sudakov form factor. For the purpose of comparison, we will consider eq. (2.26) without the $N$-dependent term (which is usually absorbed into a scale change in the parton densities)

$$
F\left(Q, q_{\mathrm{T}}\right)=Q^{2} \frac{d}{d q_{\mathrm{T}}^{2}} \int_{0}^{\infty} d \hat{b} J_{1}(\hat{b}) \exp \mathcal{S}\left(Q, \hat{b} / q_{\mathrm{T}}\right) .
$$

We will consider the following approaches:

- The full $b$-space formula at NLL, that is eq. (3.1) with $\mathcal{S}(Q, b)$ given by

$$
\mathcal{S}(Q, b)=\mathbb{S}\left(\alpha_{s}, L^{\star}\right)=\frac{1}{\alpha_{s}} f_{0}\left(\alpha_{s} L^{\star}\right)+f_{1}\left(\alpha_{s} L^{\star}\right)
$$

and

$$
L^{\star}=\log \frac{c_{2}^{2} Q^{2} b^{\star 2}}{c_{1}^{2}}, \quad b^{\star}=\frac{b}{\sqrt{1+\left(b / b_{\text {lim }}\right)^{2}}} .
$$

The functions $f_{0}$ and $f_{1}$ are given in eqs. (2.29) and (2.30). Here we choose $b_{\text {lim }}$ at the position that corresponds to the Landau pole.

- The NLL $q_{\mathrm{T}}$-space formula

$$
F\left(Q, q_{\mathrm{T}}\right)=Q^{2} \frac{d}{d q_{\mathrm{T}}^{2}}\left[\exp \mathbb{S}\left(\alpha_{s}, l\right)\left(\frac{2 c_{2}}{c_{1}}\right)^{h} \frac{\Gamma(1+h / 2)}{\Gamma(1-h / 2)}\right] .
$$

- The expression of ref. [5]. We wish to note at this point that the expression of ref. [5] differs from our eq. (3.4) by next-to-leading terms. In fact, the expression of ref. [5] is accurate at the leading-logarithmic level, with the further inclusion of subleading logarithmic terms down to the order $\alpha_{s}^{j} l^{2 j-2}$ in the expansion of the form factor. This is unlike our approach, in which terms down to the order $\alpha_{s}^{j} l^{j}$ are kept in the Sudakov exponent. 


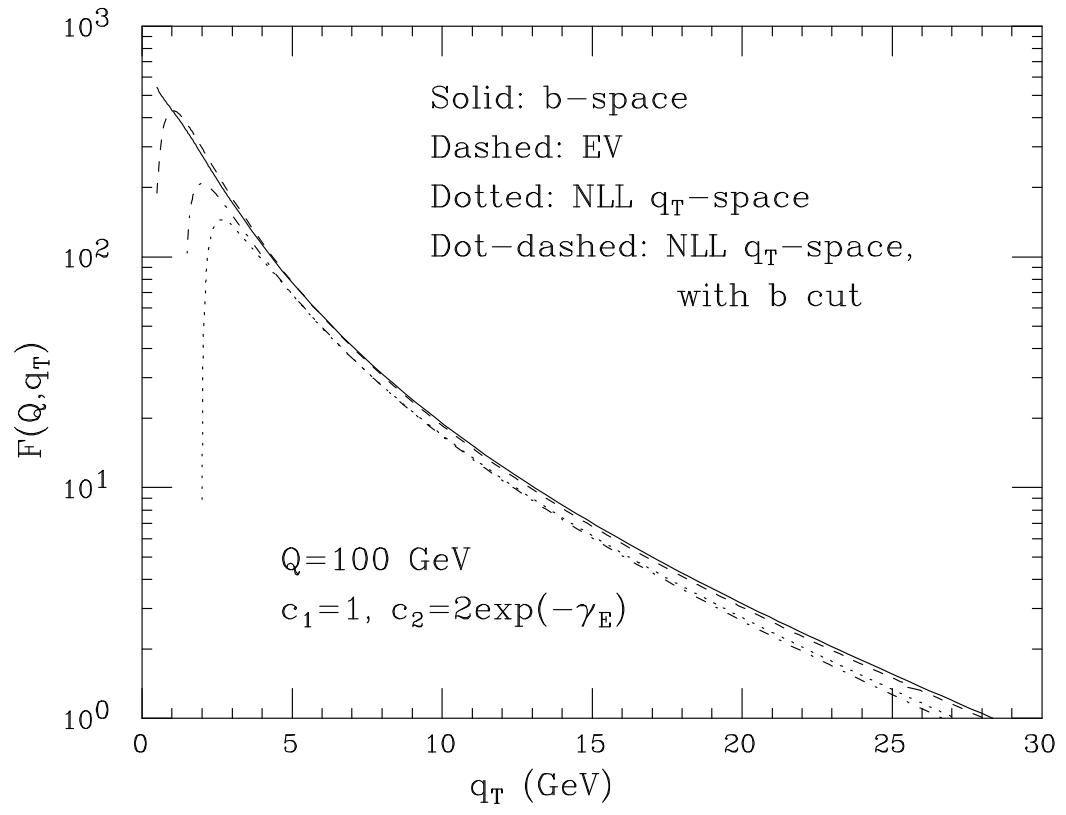

Figure 1: Comparison of the numerical results of the various approaches for the calculation of the form factor.

The results of the various approaches are summarized in fig. 1. We see that the $b$ space, the NLL $q_{\mathrm{T}}$-space, and the EV approaches are quite similar for $q_{\mathrm{T}} \geq 3 \mathrm{GeV}$. Below $3 \mathrm{GeV}$, the NLL $q_{\mathrm{T}}$-space formula begins to display a significant deviation, due to the fact that it is approaching the singularity $h \rightarrow-2$. As an illustration, we also plot a modification of the NLL $q_{\mathrm{T}^{-}}$-space formula, obtained by letting

$$
\mathcal{S}_{\mathrm{NLL}} \rightarrow \theta(b-1 / Q) \mathcal{S}_{\mathrm{NLL}}=\theta\left(\hat{b}-q_{\mathrm{T}} / Q\right) \mathcal{S}_{\mathrm{NLL}}
$$

before performing the $\hat{b}$ integration (the relevant formulae are given in Appendix B). Modified in this way, we see that the NLL result is stabilized down to smaller values of $q_{\mathrm{T}}$.

The result of ref. [5] (the dashed curve labelled EV in the figure) is better behaved at small $q_{\mathrm{T}}$, and much closer to the full $b$-space formula. In fact, the EV result differs from the $b$-space formula by next-to-leading terms. It is paradoxically the lack of these terms (which would generate the singularity) that stabilizes the result. 


\section{Conclusions}

In summary, we have examined the impact-parameter formula for the transversemomentum distribution in Drell-Yan-like processes. We have found that, when the resummed expression is expanded in powers of the strong coupling constant, and all terms suppressed by powers of $q_{\mathrm{T}} / Q$ are neglected, the coefficients of the expansion exhibit factorial growth with oscillating signs. These diverging terms arise from the small-impact-parameter region of integration.

The origin of these terms is analogous to what was found in the case of resummation of threshold-enhanced Sudakov effects [7]. In that case, however, factorially growing terms arise in the $x$-space formulation of the resummation, from the smallmomentum region of integration. No factorial growth is present in the $N$-space formulation, which is most natural in this case, since it preserves momentum conservation.

In the case we are considering here, instead, the factorial growth arises in the most natural formulation, which is the $b$-space formulation. It does, however, arise from the large-momentum region, which is unreliably treated by the resummation formalism. Presumably, its origin is due to the fact that energy constraints are not correctly implemented by the resummation formula for multigluon emission of sizeable energy.

In both cases, the factorial growth is only present in subleading (in the logarithmic sense) terms, which are not reliably given by the resummation formula.

We have obtained an analytic expression for the resummed cross section in which only the LL and NLL terms in the Sudakov exponent are retained. Thus, factorially growing terms are not present in this formula. However, the formula displays geometric singularities at finite values of $\alpha_{s} \log Q / q_{\mathrm{T}}$, which severely restrict its range of applicability. These geometric singularities arise from the small- $b$ region of integration. In the original $b$-space formula, the small- $b$ region gives rise to factorially growing terms, and thus to zero radius of convergence. This factorial growth is accompanied by sign oscillation in this formulation, and thus by strong dumping of the small- $b$ region. Thus, the formula is well defined for positive values of the coupling constant. Since, however, the factorial growth is an artefact of the $b$-space formulation, its superior stability (as far as the small- $b$ region is concerned) may hide some real problems.

We believe that a more realistic treatment of the factorization properties of mul-

tigluon emission at large transverse momenta could solve many of the problems dis- 
cussed so far, and would thus be a valuable improvement of the computation of the Drell-Yan transverse-momentum spectra.

\section{Acknowledgements}

We thank S. Catani, M. Ciafaloni, J.C. Collins, R.K. Ellis, D. Soper, G. Sterman and B.R. Webber for helpful discussions.

\section{Appendix A Coefficients of the Sudakov exponent}

In the case of DY pair production, the coefficients in eq. (1.7) are given by [10, 11]

$$
\begin{aligned}
& A_{1}=\frac{C_{\mathrm{F}}}{\pi} \\
& A_{2}=\frac{1}{\pi^{2}}\left(\frac{67}{9}-\frac{\pi^{2}}{3}-\frac{10}{27} n_{f}+\frac{8 \pi}{3} b_{0} \log \frac{c_{1} e^{\gamma_{\mathrm{E}}}}{2}\right) \\
& B_{1}=\frac{2 C_{\mathrm{F}}}{\pi} \log \frac{c_{1} e^{\gamma_{\mathrm{E}}-3 / 4}}{2 c_{2}} .
\end{aligned}
$$

The coefficient $B_{2}$ has also been computed:

$$
\begin{aligned}
B_{2}= & \frac{1}{\pi^{2}}\left[2\left(\frac{67}{9}-\frac{\pi^{2}}{3}-\frac{10}{27} n_{f}\right) \log \frac{c_{1} e^{\gamma_{\mathrm{E}}-3 / 4}}{2 c_{2}}\right. \\
& +\frac{8 \pi}{3} b_{0}\left(\log ^{2} \frac{c_{1} e^{\gamma_{\mathrm{E}}}}{2}-\log ^{2} c_{2} e^{3 / 4}\right) \\
& \left.-\frac{9}{8}+\frac{7 \pi^{2}}{6}+\frac{2 \zeta_{3}}{3}+\left(\frac{5}{36}-\frac{2 \pi^{2}}{27}\right) n_{f}\right] .
\end{aligned}
$$

However, it only contributes at the NNLL level, together with unknown contributions from the $A_{3}$ term.

\section{Appendix B $q_{\mathrm{T}}$-space result with a $b$ cut}

Our starting point is eq. (3.1), with

$$
\mathcal{S}\left(Q, \hat{b} / q_{\mathrm{T}}\right) \rightarrow \theta(\hat{b}-\rho) \mathcal{S}_{\mathrm{NLL}}\left(Q, \hat{b} / q_{\mathrm{T}}\right),
$$


where $\rho=q_{\mathrm{T}} / Q$. We obtain

$$
\begin{aligned}
F\left(Q, q_{\mathrm{T}}\right)= & Q^{2} \frac{d}{d q_{\mathrm{T}}^{2}} \int_{0}^{\infty} d \hat{b} J_{1}(\hat{b}) \exp \left[\theta(\hat{b}-\rho) \mathcal{S}_{\mathrm{NLL}}\left(Q, \hat{b} / q_{\mathrm{T}}\right)\right] \\
= & Q^{2} \frac{d}{d q_{\mathrm{T}}^{2}}\left\{\int_{0}^{\rho} d \hat{b} J_{1}(\hat{b})\left[1-\exp \mathcal{S}_{\mathrm{NLL}}\left(Q, \hat{b} / q_{\mathrm{T}}\right)\right]\right. \\
& \left.+\int_{0}^{\infty} d \hat{b} J_{1}(\hat{b}) \exp \mathcal{S}_{\mathrm{NLL}}\left(Q, \hat{b} / q_{\mathrm{T}}\right)\right\} .
\end{aligned}
$$

The second term in the curly bracket corresponds to eq. (3.4). The first term is easily computed by expanding the Bessel function

$$
J_{1}(\hat{b})=\sum_{k=1}^{\infty} C_{k} \hat{b}^{k}=\frac{\hat{b}}{2}-\frac{\hat{b}^{3}}{16}+\ldots
$$

We obtain

$$
\begin{aligned}
F\left(Q, q_{\mathrm{T}}\right)= & Q^{2} \frac{d}{d q_{\mathrm{T}}^{2}}\left\{\sum_{k=1}^{\infty} C_{k}\left[\frac{\rho^{k+1}}{k+1}-\exp \mathbb{S}\left(\alpha_{s}, l\right)\left(\frac{c_{2}}{c_{1}}\right)^{h} \frac{\rho^{h+k+1}}{h+k+1}\right]\right. \\
& \left.+\exp \mathbb{S}\left(\alpha_{s}, l\right)\left(\frac{2 c_{2}}{c_{1}}\right)^{h} \frac{\Gamma(1+h / 2)}{\Gamma(1-h / 2)}\right\} .
\end{aligned}
$$

Notice that the term with $k=1$ in the sum has a singularity for $h=-2$, which cancels exactly the analogous singularity arising in the second term from $\Gamma(1+h / 2)$. Similarly, the singularities for $h=-4,-6, \ldots$ are cancelled by the higher-order terms in the sum.

\section{Appendix C Growth of the resummed expansion}

In this Appendix, we show that the large- $\hat{b}$ region of the integral

$$
\int_{\hat{b}_{0}}^{\infty} d \hat{b} J_{1}(\hat{b}) I\left(\alpha_{s}, L\right)
$$

with

$$
I\left(\alpha_{s}, L\right)=\exp \left\{\frac{1}{\alpha_{s} b_{0}^{2}}\left[\alpha_{s} b_{0} L+\log \left(1-\alpha_{s} b_{0} L\right)\right]\right\}
$$

does not generate factorially growing coefficients in the perturbative expansion. 
In the following, we will use the notation

$$
\sum_{j, k} C_{j, k}\left(-\alpha_{s}\right)^{j}(-L)^{k} \leq \sum_{j, k} G_{j, k}\left(-\alpha_{s}\right)^{j}(-L)^{k}
$$

to mean that

$$
\left|C_{j, k}\right| \leq\left|G_{j, k}\right|
$$

for all values of $j$ and $k$. The minus sign in front of $\alpha_{s}$ and $L$ above is actually irrelevant, and it is there only to make the following discussion more transparent. We have

$$
I\left(\alpha_{s}, L\right)=\exp \frac{\alpha_{s} b_{0} L+\log \left(1-\alpha_{s} b_{0} L\right)}{\alpha_{s} b_{0}^{2}}=\exp \sum_{k=2}^{\infty} \frac{b_{0}^{k-1}\left(-\alpha_{s}\right)^{k-1}(-L)^{k}}{b_{0} k} .
$$

The coefficients of $\left(-\alpha_{s}\right)^{k-1}(-L)^{k}$ in the power series in the exponent are all positive, and the exponential function has a power expansion with positive coefficients. Thus, if we increase the coefficients of $\left(-\alpha_{s}\right)^{k-1}(-L)^{k}$ in the exponent, we will surely obtain an expression that is larger (in the sense of eq. (C.4)) than the original one. We thus replace $k \rightarrow k-1$ in the denominators of the coefficients, and obtain

$$
I\left(\alpha_{s}, L\right) \leq \exp \sum_{k=2}^{\infty} \frac{b_{0}^{k-1}\left(-\alpha_{s}\right)^{k-1}(-L)^{k}}{b_{0}(k-1)}=\left(1-\alpha_{s} b_{0} L\right)^{y} .
$$

where $y=L / b_{0}$. We now continue with the trivial inequalities

$$
\begin{aligned}
I\left(\alpha_{s}, L\right) & \leq\left(1-\alpha_{s} b_{0} L\right)^{y} \\
& =\sum_{k=0}^{\infty}\left(-\alpha_{s} b_{0} L\right)^{k} \frac{y(y-1) \ldots(y-k+1)}{k !} \\
& \leq \sum_{k=0}^{\infty}\left(\alpha_{s} b_{0} L\right)^{k} \frac{(y+k)^{k}}{k^{k} 2^{-k}} \leq \sum_{k=0}^{\infty}\left(2 \alpha_{s} b_{0}^{2}\right)^{k}(y+1)^{2 k},
\end{aligned}
$$

where we have used the property

$$
k !>k^{k} 2^{-k} .
$$

We thus have to examine the asymptotic behaviour of the integral

$$
\int_{\hat{b}_{0}}^{\infty} d \hat{b} J_{1}(\hat{b})(y+1)^{2 k},
$$


which in turn is that of an integral of the form ${ }^{8}$

$$
\int_{\hat{b}_{0}}^{\infty} d \hat{b} \exp (-\hat{b}) \log ^{2 k} \hat{b}
$$

Using saddle-point methods, this is easily seen to grow like $\log ^{2 k} 2 k$, which is stronger than geometric, but much less than factorial.

\section{Appendix D A few remarks on the non-perturbative part}

We consider here the $b$-space resummation expression

$$
\begin{aligned}
& \frac{d \sigma}{d Q^{2} d q_{\mathrm{T}}^{2}}=\frac{\sigma_{0}}{Q^{2}} \sum_{q} e_{q}^{2} \int d x_{\mathrm{A}} d x_{\mathrm{B}} \delta\left(x_{\mathrm{A}} x_{\mathrm{B}}-\tau\right) \int d^{2} b e^{i \vec{q}_{\mathrm{T}} \cdot \vec{b}} \\
& \quad \times\left\{f_{q / \mathrm{A}}\left(x_{\mathrm{A}}, c_{1} / b^{\star}\right) f_{\bar{q} / \mathrm{B}}\left(x_{\mathrm{B}}, c_{1} / b^{\star}\right) \exp \mathcal{S}\left(Q, b^{\star}\right)+(q \leftrightarrow \bar{q})\right\},
\end{aligned}
$$

where

$$
b^{\star}=\frac{b}{\sqrt{1+b^{2} / b_{\lim }^{2}}} .
$$

The new variable $b^{\star}$ is approximately equal to $b$ for small $b$, but never exceeds $b_{\text {lim }}$. We should have $b_{\lim } \lesssim 1 / \Lambda_{\mathrm{QCD}}$, so that both the Sudakov exponent (1.6) and the parton densities do not become undefined because of the Landau pole.

The region of large $b$ is in any case out of the control of perturbation theory, since it involves large values of the strong coupling constant. Therefore, one might want to keep $b_{\text {lim }}$ substantially smaller than the value corresponding to the Landau pole, and parametrize non-perturbative effects by modifying the form factor in a way that leaves it unaffected at small $b$. A procedure that is often adopted is to multiply the integrand of eq. (2.3) by

$$
F_{N P}=e^{-g b^{2}}
$$

where the parameter $g$ can be adjusted to the data. Notice that at large $b, b^{\star}$ approaches $b_{\text {lim }}$, and therefore $\exp \mathcal{S}$ approaches a constant value while $\exp \left(i b q_{\mathrm{T}}\right)$ oscillates. Nevertheless, the integral is convergent even in the limit $g \rightarrow 0$. In fact, if we add and subtract the contribution of $\exp \mathcal{S}$ at $b=b_{\text {lim }}$ we obtain

$$
F\left(Q, q_{\mathrm{T}}\right)=\frac{Q^{2}}{4 \pi} \int d^{2} b e^{i \vec{q}_{\mathrm{T}} \cdot \vec{b}} e^{-g b^{2}}\left[\exp \mathcal{S}\left(Q, b^{\star}\right)-\exp \mathcal{S}\left(Q, b_{\lim }\right)\right]
$$

\footnotetext{
${ }^{8}$ Here we use the same method as was used in eq. (2.13), and also use the fact that only the large- $\hat{b}$ region can lead to stronger-than-geometric growth.
} 


$$
+\frac{Q^{2}}{4 g} e^{-q_{\mathrm{T}}^{2} /(4 g)} \exp \mathcal{S}\left(Q, b_{\mathrm{lim}}\right) .
$$

For $g=0$ the integral is now convergent at $b \rightarrow+\infty$ for any choice of $b_{\text {lim }}$, and for the remaining term we have

$$
\lim _{g \rightarrow 0} \frac{Q^{2}}{4 g} e^{-q_{\mathrm{T}}^{2} /(4 g)} \exp \mathcal{S}\left(Q, b_{\text {lim }}\right)=Q^{2} \pi \delta^{2}\left(\vec{q}_{\mathrm{T}}\right) \exp \mathcal{S}\left(Q, b_{\text {lim }}\right) .
$$

This fact may be useful to assess the relevance of the non-perturbative term compared to the effects of resummation.

\section{References}

[1] Yu.L. Dokshitzer, D.I. Dyakonov and S.I. Troyan, Phys. Rep. 58(1980)269.

[2] G. Parisi and R. Petronzio, Nucl. Phys. B154(1979)427.

[3] J.C. Collins, D. Soper and G. Sterman, Nucl. Phys. B250(1985)199.

[4] J.C. Collins and D.E. Soper, Nucl. Phys. B197(1982)446.

[5] R.K. Ellis and S. Veseli, Nucl. Phys. B511(1998)649, hep-ph/9706526.

[6] R.K. Ellis, D.A. Ross and S. Veseli, Nucl. Phys. B503(1997)309, hep-ph/9704239.

[7] S. Catani, M. Mangano, P. Nason and L. Trentadue, Nucl. Phys. B478(1996)273, hep-ph/9604351.

[8] P.E.L. Rakow and B.R. Webber, Nucl. Phys. B187(1981)254.

[9] G. Turnock, preprint CAVENDISH-HEP-92-3 (1992).

[10] C. Davies, B.R. Webber and W.J. Stirling, Nucl. Phys. B256(1985)413.

[11] J. Kodaira and L. Trentadue, Phys. Lett. B112(1982)66;

C.T.H. Davies and W.J. Stirling, Nucl. Phys. B244(1984)337;

R.K. Ellis, G. Martinelli and R. Petronzio, Phys. Lett. B104(1981)45, Nucl. Phys. B211(1983)106. 